\title{
A!
}

This is an electronic reprint of the original article.

This reprint may differ from the original in pagination and typographic detail.

Rajamäki, Robin; Koivunen, Visa

\section{Sparse Active Rectangular Array with Few Closely Spaced Elements}

\author{
Published in: \\ IEEE Signal Processing Letters
}

DOI:

10.1109/LSP.2018.2876066

Published: 01/01/2018

Document Version

Peer reviewed version

Please cite the original version:

Rajamäki, R., \& Koivunen, V. (2018). Sparse Active Rectangular Array with Few Closely Spaced Elements. IEEE Signal Processing Letters, 25(12), 1820-1824. https://doi.org/10.1109/LSP.2018.2876066

This material is protected by copyright and other intellectual property rights, and duplication or sale of all or part of any of the repository collections is not permitted, except that material may be duplicated by you for your research use or educational purposes in electronic or print form. You must obtain permission for any other use. Electronic or print copies may not be offered, whether for sale or otherwise to anyone who is not an authorised user. 


\title{
Sparse Active Rectangular Array with Few Closely Spaced Elements
}

\author{
Robin Rajamäki, Student Member, IEEE, and Visa Koivunen, Fellow, IEEE
}

\begin{abstract}
Sparse sensor arrays offer a cost effective alternative to uniform arrays. By utilizing the co-array, a sparse array can match the performance of a filled array, despite having significantly fewer sensors. However, even sparse arrays can have many closely spaced elements, which may deteriorate the array performance in the presence of mutual coupling. This paper proposes a novel sparse planar array configuration with few unit inter-element spacings. This Concentric Rectangular Array (CRA) is designed for active sensing tasks, such as microwave or ultrasound imaging, in which the same elements are used for both transmission and reception. The properties of the CRA are compared to two well-known sparse geometries: the Boundary Array and the Minimum-Redundancy Array (MRA). Numerical searches reveal that the CRA is the MRA with the fewest unit element displacements for certain array dimensions.
\end{abstract}

Keywords-Sparse array, sum co-array, mutual coupling

\section{INTRODUCTION}

The number of antenna elements and RF front ends are critical cost factors in phased sensor arrays. Especially uniform planar arrays rapidly become expensive with increasing array dimensions. Fortunately, sparse arrays exploiting the co-array [1] may to a certain extent match the performance of uniform arrays, using only a fraction of the number of elements. The co-array is a virtual structure arising from the sums or differences of physical element pairs. It determines, for example, the number of sources an array can resolve [2]-[6], or the achievable point spread function (PSF) in array imaging [7]. Many applications, such as radar and medical ultrasound, can take advantage of the co-array in order to achieve a desired PSF using fewer sensors. Another benefit of sparse arrays is that they have fewer closely spaced elements. This may reduce mutual coupling [8]-[10], whose magnitude is inversely proportional to the inter-element distance [11], [12].

The objective of sparse array design is often to find the array configuration that generates a desired co-array using as few elements as possible. Unfortunately, the resulting optimization problem is combinatorial and difficult to solve exactly, even for modest array sizes. Hence, several authors have investigated closed-form, but possibly sub-optimal configurations, such as the Wichmann [13]-[15], Nested [3], or Co-prime array [16]. These arrays have primarily been developed for passive sensing of incoherent sources, although some of them are easily adapted to active array processing tasks, such as coherent imaging with co-located transceivers [17], [18]. Note that optimal active sparse linear arrays with distinct transmitting and

The authors are with the Department of Signal Processing and Acoustics, Aalto University School of Electrical Engineering, Espoo 02150, Finland (email: robin.rajamaki@aalto.fi, visa.koivunen@aalto.fi). receiving elements are straightforward to generate using interpolation [19]. In the case of planar arrays, placing elements on a convex boundary is equivalent to filling the interior of the array with virtual elements [20]. This Boundary Array (BA) [7] has been shown to be optimal with respect to the number of elements in some cases [21]. However, many of the elements in the BA are closely spaced, which may be problematic in the face of non-negligible mutual coupling. Consequently, a desirable design goal could be to increase physical element displacements without changing the co-array support [9], [10].

This paper proposes a novel planar sparse array configuration for active sensing called the Concentric Rectangular Array (CRA). The CRA has the same number of sensors as the BA, but the number of elements separated by the smallest distance (typically half a wavelength) is practically constant, whereas it grows linearly with aperture for the BA. We proove that the CRA has both a contiguous difference and sum co-array, which allows the array to achieve comparable performance with a filled array of equivalent aperture in both passive and active sensing. More generally, we show that any mirror symmetric array, such as the CRA, has an equivalent difference and sum co-array. The CRA is also verified to be minimally redundant for certain square arrays. For non-square rectangular arrays, the CRA trades off redundancy for fewer closely spaced elements.

The paper is organized as follows: section II introduces the signal model and definitions. Section III presents the CRA and establishes its key properties. A numerical imaging example is provided in section IV, and section $\mathrm{V}$ concludes the paper.

An interval of integers with step size $m$ is denoted $\{a: m$ : $b\}=\{a, a+m, \ldots, b\}$, where brackets denote a set. Shorthand $\{a: b\}$ is used when $m=1$. The Cartesian product of onedimensional sets $\mathcal{A}$ and $\mathcal{B}$ is $\mathcal{A} \times \mathcal{B}=\left\{[a b]^{\mathrm{T}} \mid a \in \mathcal{A} ; b \in \mathcal{B}\right\}$.

\section{Signal MOdEL AND DEFINITIONS}

Consider an active planar array with $N$ co-located, singlemode [22] transmitting (Tx) and receiving ( $\mathrm{Rx}$ ) elements illuminating $K$ far field point targets with narrowband radiation. The transmitters are operated sequentially within the coherence time of the scene, allowing a noise-free snapshot to be modeled by the natural data matrix [2] $\mathbf{X} \in \mathbb{C}^{N \times N}$, whose rows correspond to the Rx and columns to the Tx elements:

$$
\mathbf{X}=\mathbf{M}_{\mathrm{r}} \mathbf{A}_{\mathrm{r}} \boldsymbol{\Gamma} \mathbf{A}_{\mathrm{t}}^{\mathrm{T}} \mathbf{M}_{\mathrm{t}}^{\mathrm{T}} .
$$

In (1), $\boldsymbol{\Gamma}=\operatorname{diag}\left(\left[\gamma_{1} \ldots \gamma_{K}\right]\right)$ is a diagonal matrix containing the target reflectivities. Furthermore, $\mathbf{M}_{\mathrm{r}}, \mathbf{M}_{\mathrm{t}} \in \mathbb{C}^{N \times N}$ are the $\mathrm{Rx}$ and Tx mutual coupling matrices, and $\mathbf{A}_{\mathrm{r}}, \mathbf{A}_{\mathrm{t}} \in \mathbb{C}^{N \times K}$ the respective steering matrices. If the elements are reciprocal, then $\mathbf{M}_{\mathrm{r}}=\mathbf{M}_{\mathrm{t}}=\mathbf{M}$ and $\mathbf{A}_{\mathrm{r}}=\mathbf{A}_{\mathrm{t}}=\mathbf{A}$. In case of negligible mutual 
coupling $(\mathbf{M} \approx \mathbf{I})$ and identical omnidirectional elements $\left(A_{n k}=e^{j 2 \pi \mathbf{v}_{k}^{\mathrm{T}} \mathbf{d}_{n} / \lambda}\right)$, the $(n, m)^{\text {th }}$ element of $\mathbf{X}$ in (1) assumes the form: $X_{n m}=\sum_{k=1}^{K} \gamma_{k} e^{j 2 \pi \mathbf{v}_{k}^{\mathrm{T}}\left(\mathbf{d}_{n}+\mathbf{d}_{m}\right) / \lambda}$. Here $\lambda$ is the carrier wavelength, and $\mathbf{v}_{k}=\left[\begin{array}{ll}\sin \left(\varphi_{k}\right) \sin \left(\theta_{k}\right) \cos \left(\varphi_{k}\right) \sin \left(\theta_{k}\right)\end{array}\right]^{\mathrm{T}}$ the direction of the $k^{\text {th }}$ target with azimuth $\varphi_{k} \in[-\pi, \pi]$ and elevation $\theta_{k} \in[0,2 \pi]$. Element positions are given by set $\mathcal{D}=\left\{\mathbf{d}_{n} \in \mathbb{R}^{2}\right\}_{n=1}^{N}$, and term $\mathbf{d}_{n}+\mathbf{d}_{m}$ represents an element of the sum co-array.

\section{A. Co-array}

The sum co-array is a virtual array formed by pairwise vector sums of $\mathrm{Tx}$ and $\mathrm{Rx}$ elements: $\mathcal{C}_{\Sigma}=\mathcal{D}+\mathcal{D}=\left\{\mathbf{d}_{\Sigma}=\right.$ $\left.\mathbf{d}_{m}+\mathbf{d}_{n} \mid \mathbf{d}_{n}, \mathbf{d}_{m} \in \mathcal{D}\right\}$. Similarly, subtraction give rise to the difference co-array: $\mathcal{C}_{\Delta}=\mathcal{D}-\mathcal{D}$. Dedicated array processing algorithms, such as image addition [7] or spatial smoothing MUSIC [3], are required to fully utilize the co-array. The coarray is also characterized by the multiplicity of each element. In case of the difference co-array, the multiplicity function is $v_{\Delta}\left(\mathbf{d}_{\Delta}\right)=\sum_{\mathbf{d}_{n}, \mathbf{d}_{m} \in \mathcal{D}} \mathbb{1}\left(\mathbf{d}_{\Delta}=\mathbf{d}_{m}-\mathbf{d}_{n}\right)$, where $\mathbb{1}(\cdot)$ is the indicator function. For simplicity, physical elements are usually assumed to lie on a uniform grid, which after normalization by the unit inter-element spacing simplifies to set of integervalued vectors contained within an $L_{x} \times L_{y}$ rectangle, i.e., $\mathcal{D}=$ $\left\{\left[\begin{array}{ll}d_{x} & d_{y}\end{array}\right]^{\mathrm{T}} \in \mathbb{N}^{2} \mid 0 \leq d_{x} \leq L_{x} ; 0 \leq d_{y} \leq L_{y}\right\}$. A unit on this grid typically corresponds to a physical distance of $\lambda / 2$. Since $\mathcal{D} \subseteq\left\{0: L_{x}\right\} \times\left\{0: L_{y}\right\}$ it follows that $\mathcal{C}_{\Sigma} \subseteq\left\{0: 2 L_{x}\right\} \times\left\{0: 2 L_{y}\right\}$, and $\mathcal{C}_{\Delta} \subseteq\left\{-L_{x}, L_{x}\right\} \times\left\{-L_{y}, L_{y}\right\}$. The sum and difference co-array are contiguous when $\mathcal{C}_{\Sigma}=\left\{0: 2 L_{x}\right\} \times\left\{0: 2 L_{y}\right\}$ and $\mathcal{C}_{\Delta}=\left\{-L_{x}, L_{x}\right\} \times\left\{-L_{y}, L_{y}\right\}$. A contiguous co-array maximizes the number of virtual elements for a given aperture.

Next, it is shown that a symmetric array with a contiguous sum co-array implies a contiguous difference co-array, and vice versa. In fact, the multiplicity functions of the difference and sum co-array are equal up to a shift of the support:

Lemma 1 (Co-array of symmetric array): If $\mathcal{D}$ is mirror symmetric, then $v_{\Sigma}(\mathcal{D}+\mathcal{D})=v_{\Delta}(\mathcal{D}-\mathcal{D})$.

Proof: This follows from the equivalence of the convolution and autocorrelation of a real symmetric function. For simplicity, consider the one-dimensional case, which is then straightforward to generalize to higher dimensions. Let $b[n]=\mathbb{1}(n=d), n \in \mathbb{Z}$ be a binary sequence indicating the element positions $d \in \mathcal{D}$. The multiplicity functions are given by the convolution $v_{\Sigma}[n]=(b * b)[n]=\sum_{m=0}^{L} b[m] b[n-m]$, and the autocorrelation $v_{\Delta}[n]=(b \star b)[n]=\sum_{m=0}^{L} b^{*}[m] b[n+m]$. Since $b$ is real and symmetric; $v_{\Sigma}[n]=0, n \notin\{0: 2 L\}$; and $v_{\Delta}[n]=0, n \notin\{-L: L\}$, it follows that $v_{\Delta}[n-L]=$ $\sum_{m=0}^{L} b[m] b[n+m-L]=\sum_{m=0}^{L} b[m] b[n-m]=v_{\Sigma}[n]$, i.e., $v_{\Delta}\left(\mathcal{C}_{\Delta}\right)=v_{\Delta}\left(\mathcal{C}_{\Sigma}-L\right)=v_{\Sigma}\left(\mathcal{C}_{\Sigma}\right)$.

The ideal assumptions of no mutual coupling, far field targets, and narrowband signals are key to illustrating the emergence of the co-array. Obviously these assumptions hold only approximately in practice. Moreover, practical arrays are subject to other non-idealities, such as imprecise knowledge of elements' phase centers, which introduce perturbations into the array manifold and co-array [23]. The co-array is nevertheless a useful concept that can be exploited in real-world array processing tasks [24]-[27].

\section{B. Employed figures of merit for arrays}

1) Redundancy, $R$, quantifies the degree of element repetition in the co-array. A non-redundant array achieves $R=$ 1. Typically, $R>1$ for an array with a contiguous coarray. In case of the sum co-array, redundancy is defined $R=N(N+1) /\left(2\left|\mathcal{C}_{\Sigma}\right|\right)$ [28]. Furthermore, the asymptotic redundancy $R_{\infty}=\lim _{N \rightarrow \infty} R$ is often used to compare sparse array configurations. For example, any rectangular array with a contiguous sum co-array must satsify $R_{\infty} \geq 1.19$ [29].

2) Sparseness, $S$, counts the number of element pairs separated by $d>0$, i.e., $S(d)=\frac{1}{2} \sum_{\mathbf{d}_{\Delta} \in \mathcal{C}_{\Delta}} v_{\Delta}\left(\mathbf{d}_{\Delta}\right) \cdot \mathbb{1}\left(\left\|\mathbf{d}_{\Delta}\right\|_{2}=d\right)$. When $\mathcal{C}_{\Delta} \subseteq \mathbb{Z}^{2}$, then $d=1, \sqrt{2}, 2, \sqrt{5}, \sqrt{8}, 3, \ldots$, and $S(1)$ is the number of unit spacings. For linear arrays $S(d)=v_{\Delta}(d)$.

\section{Array configurations}

Next, three well-known co-array equivalent array configurations with contiguous sum co-arrays are reviewed. First however, a useful and easily verifiable property of any planar array with a contiguous sum co-array is stated. Namely, the corners of the array must contain at least three elements each:

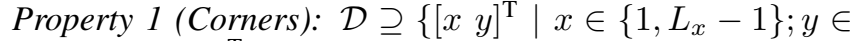
$\left.\left\{0, L_{y}\right\}\right\} \cup\left\{\left[\begin{array}{ll}x & y\end{array}\right]^{\mathrm{T}} \mid x \in\left\{0, L_{x}\right\} ; y \in\left\{0,1, L_{y}-1, L_{y}\right\}\right\}$.

1) The Uniform Rectangular Array (URA) is a planar array, whose elements cover the rectangle $\mathcal{D}=\left\{0: L_{x}\right\} \times\left\{0: L_{y}\right\}$. The URA has a simple and periodic, but highly redundant structure.

2) The Minimum-Redundancy Array (MRA) minimizes the number of elements subject to a contiguous co-array [28], [30]. MRAs with a rectangular sum co-array solve: $\operatorname{minimize}_{\mathcal{D}}|\mathcal{D}|$ s.t. $\mathcal{D}+\mathcal{D}=\left\{0: 2 L_{x}\right\} \times\left\{0: 2 L_{y}\right\}$. In general, this is a difficult non-convex problem with several feasible solutions. For example, when $L_{x}=L_{y}=L=12$ the MRA has $N=52$ elements, which yields $\mathcal{O}\left(10^{44}\right)$ possible configurations of which 27108 are feasible solutions [21]. Nevertheless, planar MRAs have been found for $L_{x}, L_{y} \leq 13$ in [21] using a combinatorial algorithm [31], which combines component solutions found using a branch-and-bound algorithm with dynamic pruning [32]. Moreover, the solution nonuniqueness issue may be overcome by regularization. In this paper, sparseness $S(1)$ is minimized among feasible MRAs, yielding the MRA with the fewest unit element displacements.

3) The Boundary Array (BA) [20], [24] consists of a hollow perimeter of elements. Actually, a BA of any convex shape has a contiguous sum and difference co-array [20]. The BA has empirically been found to be an MRA for square arrays with $L \leq 23$ [21]. However, the BA still has many closely spaced elements, due to the uniform linear arrays on its boundaries.

\section{CONCENTRIC RECTANGULAR ARRAY}

Definition 1: For even $L_{x}, L_{y} \geq 2$, the elements of the Concentric Rectangular Array (CRA) are given by:

$$
\begin{aligned}
\mathcal{D}_{\mathrm{CRA}}=\bigcup_{i=0}^{2}\left\{\left[d_{x}, d_{y}\right]^{\mathrm{T}} \mid d_{x} \in \mathcal{D}_{i}\left(L_{x}\right) ; d_{y} \in\left\{i, L_{y}-i\right\}\right\} \\
\cup\left\{\left[d_{x}, d_{y}\right]^{\mathrm{T}} \mid d_{x} \in\left\{i, L_{x}-i\right\} ; d_{y} \in \mathcal{D}_{i}\left(L_{y}\right)\right\},
\end{aligned}
$$

where $\mathcal{D}_{0}(L)=\{0, L\} \cup\{1: 2: L-1\}, \mathcal{D}_{1}(L)=\{0,1, L-$ $1, L\}, \mathcal{D}_{2}(L)=\{2: 2: L-2\}$. 

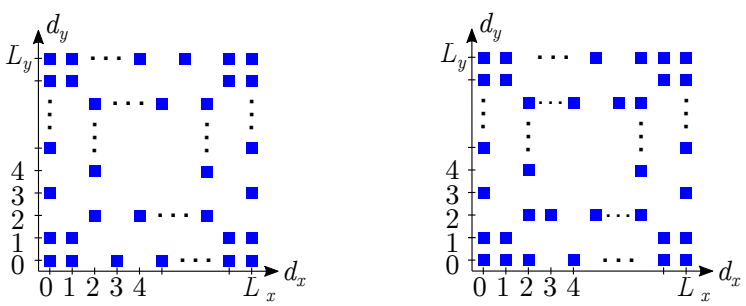

Fig. 1. CRA for even $L_{x}, L_{y}$ (left), and odd $L_{x}$, even $L_{y}$ (right).

The CRA may also be constructed for odd $L_{x}, L_{y}$ with the minor modifications illustrated in Fig. 1. Essentially, the CRA consists of two sparse interleaved coaxial rectangles displaced by two unit spacings. This structure ensures that the array only has a few closely spaced elements, and that the sum co-array is contiguous, as shown by the following theorem:

Theorem 1 (Contiguous sum co-array): $\mathcal{C}_{\Sigma, \mathrm{CRA}}=\mathcal{D}_{\mathrm{CRA}}+$ $\mathcal{D}_{\mathrm{CRA}}=\left\{0: 2 L_{x}\right\} \times\left\{0: 2 L_{y}\right\}$.

Proof: The theorem is proved for even $L_{x}, L_{y}$. The proof for odd dimensions is similar and hence omitted. Assume $L_{x}=$ $L_{y}=L$ without loss of generality. Let $\mathcal{D}_{l}(L)=\mathcal{D}_{l}$ denote the set of one-dimensional element coordinates on row $l$. When $l \leq L$, the $l^{\text {th }}$ row of the co-array is $\mathcal{C}_{l}=\bigcup_{i=0}^{\lfloor l / 2\rfloor} \mathcal{D}_{i}+\mathcal{D}_{l-i}$, which in case of the CRA simplifies to $\mathcal{C}_{l}=\left(\mathcal{D}_{0}+\mathcal{D}_{l}\right) \cup\left(\mathcal{D}_{1}+\right.$ $\left.\mathcal{D}_{l-1}\right) \cup\left(\mathcal{D}_{2}+\mathcal{D}_{l-2}\right)$. For $l=0$ and $l=1, \mathcal{C}_{0}=\mathcal{D}_{0}+\mathcal{D}_{0} \supseteq$ $\mathcal{C}_{1}=\mathcal{D}_{0}+\mathcal{D}_{1}=\{0,1,3, \ldots, L-1, L\}+\{0,1, L-1, L\}=$ $\{0: 2 L\}$. Similarly, $\mathcal{C}_{2}=\left(\mathcal{D}_{0}+\mathcal{D}_{2}\right) \cup\left(\mathcal{D}_{1}+\mathcal{D}_{1}\right) \supseteq(\{2:$ $L-1\} \cup\{L+1: 2 L-2\}) \cup\{0,1, L, 2 L-1,2 L\}=\{0: 2 L\}$. When $l \geq 3$ and odd, $\mathcal{C}_{l} \supseteq\left(\mathcal{D}_{0}+\mathcal{D}_{l}\right) \cup\left(\mathcal{D}_{2}+\mathcal{D}_{l-2}\right) \supseteq$ $(\{0,2 L\} \cup\{1: 2: 2 L-1\}) \cup\{2: 2: 2 L-2\}=\{0: 2 L\}$. When $l \geq 4$ and even, $\mathcal{C}_{l} \supseteq\left(\mathcal{D}_{0}+\mathcal{D}_{l}\right) \cup\left(\mathcal{D}_{1}+\mathcal{D}_{l-1}\right) \cup$ $\left(\mathcal{D}_{2}+\mathcal{D}_{l-2}\right) \supseteq(\{3: 2: 2 L-3\} \cup\{2,2 L-2\}) \cup\{4:$ $2: 2 L-4\} \cup\{0,1,2 L-1,2 L\}=\{0: 2 L\}$. Consequently, $\mathcal{C}_{\Sigma} \supseteq\{0: 2 L\} \times\{0: L\}$. Due to symmetry, $\mathcal{C}_{l}=\{0: 2 L\}$ when $L+1 \leq l \leq 2 L$, yielding $\mathcal{C}_{\Sigma}=\{0: 2 L\} \times\{0: 2 L\}$.

Similarly to the BA and URA, the CRA also has a contiguous difference co-array, as stated in the following corollary:

Corollary 1 (Contiguous difference co-array): $\mathcal{C}_{\Delta, \mathrm{CRA}}=$ $\mathcal{D}_{\mathrm{CRA}}-\mathcal{D}_{\mathrm{CRA}}=\left\{-L_{x}: L_{x}\right\} \times\left\{-L_{y}: L_{y}\right\}$.

Proof: This follows from Lemma 1 and Theorem 1.

\section{A. Comparison with other array configurations}

Next, the CRA is compared to the BA, MRA, and URA in terms of redundancy and sparseness. Results are summarized in Table I. Note that only configurations with a contiguous sum co-array are considered. E.g., the Hourglass Array [10] is sparser than the CRA, but its sum co-array has holes.

1) Number of elements and redundancy: For even $L_{x}, L_{y} \geq$ 6 , the CRA has the same number of elements as the BA, i.e., $N=2\left(L_{x}+L_{y}\right)$. Recall that the BA is an MRA when $L_{x}=L_{y}=L \leq 23$. Fig. 2 shows the redundancy of the three configurations for $L \in\{0: 20\}$. The redundancy of the CRA equals that of the BA and MRA for even $L \geq 6$. For odd $L$, the CRA has two more elements than the BA. It is straightforward to show that all elements of the CRA are essential [33], i.e., removing a sensor introduces a hole in the sum co-array (but not necessarily the difference co-array). When $L=\{6: 2: 23\}$, this follows directly from the MRA property of the CRA.

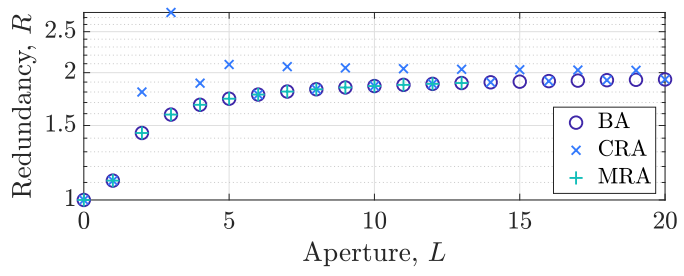

Fig. 2. Redundancy of sparse square arrays. The CRA and BA are equally redundant for even apertures $L \geq 6$. These arrays are also MRAs when $L \leq 23$ [21]. MRAs for $L \geq 14$ have not yet been exhaustively listed [21].

2) Sparseness: Fig. 1 shows that the number of smallest inter-element distances in the CRA is practically independent of the array dimensions. Specifically, $16 \leq S(1) \leq 20$ and $12 \leq S(\sqrt{2}) \leq 16$, when $L_{x}, L_{y} \geq 4$. This is a significant improvement over the BA with $S(1)=\mathcal{O}\left(L_{x}+L_{y}\right)$, and the URA with $S(1)=\mathcal{O}\left(L_{x} L_{y}\right)$. In fact, Property 1 guarantees that the CRA has at most 8 more unit spacings than any sum co-array equivalent array, including the MRA. Fig. 3 shows that although the CRA has two extra elements compared to the MRA when $L=13$, the CRA still has lower $S(1)$ (Fig. 4). Moreover, an exhaustive search of MRAs [21] reveals that the CRA is the MRA with the lowest $S(1)$ when $L \in\{6: 2: 12\}$.
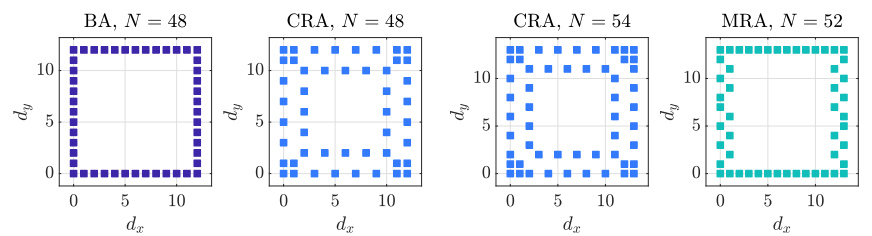

Fig. 3. Sparse square arrays. Left: BA and CRA for even aperture $L=12$. The CRA is numerically found to be the MRA with fewest unit spacings for $L \in\{6,8,10,12\}$. Right: CRA and MRA for odd $L=13$. The CRA has fewer closely spaced elements, despite having two more elements than the MRA.

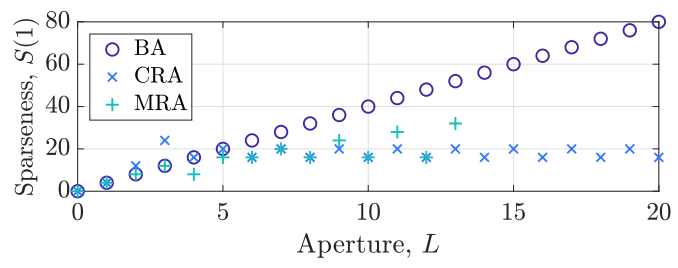

Fig. 4. Number of unit spacings in square arrays. The CRA has more elements than the MRA for $L \in\{9,11,13\}$, but fewer of them are closely spaced.

3) Asymptotic redundancy: The array aspect ratio is defined $\rho=\left(L_{y}+1\right) /\left(L_{x}+1\right) \in(0,1]$, where $L_{x} \geq L_{y}$ is assumed without loss of generality. After simple manipulations, the asymptotic redundancy (section II-B1) of the CRA and BA becomes: $R_{\infty}=(\rho+1)^{2} /(2 \rho)$. This expression achieves its minimum value $R_{\infty}=2$ at $\rho=1$, which corresponds to the square array. For other aspect ratios, $R_{\infty}>2$. Recently, two configurations achieving a constant asymptotic redundancy for 
TABLE I. PROPERTIES OF ARRAY CONFIGURATIONS AS A FUNCTION OF Dimensions $L_{x} \times L_{y}$ AND ASPECT RATIO $\rho=\left(L_{y}+1\right) /\left(L_{y}+1\right)$.

\begin{tabular}{|c|c|c|c|c|c|}
\hline Array configuration & No. of elements, $N$ & Sparseness, $S(1)$ & $S(\sqrt{2})$ & $S(2)$ & Asymptotic redundancy, $R_{\infty}$ \\
\hline Uniform Rectangular Array (URA) & $L_{x} L_{y}$ & $2 L_{x} L_{y}+L_{x}+L_{y}$ & $2 L_{x} L_{y}$ & $2 L_{x} L_{y}-L_{x}-L_{y}$ \\
Minimum-Redundancy Array (MRA) & N/A & $\geq 8$ & $\geq 2$ & $\geq 1$ \\
Boundary Array (BA) [20] & $2\left(L_{x}+L_{y}\right)$ & $2\left(L_{x}+L_{y}\right)$ & 4 & $2\left(L_{x}+L_{y}\right)-4$ \\
Concentric Rectangular Array (CRA) & $2\left(L_{x}+L_{y}\right)$ & 16 & 12 & $2\left(L_{x}+L_{y}\right)-12$ & $(\rho+1)^{2} /(2 \rho)$ \\
\end{tabular}

any aspect ratio were introduced: the Dense-Sparse and Short Bars Array [21]. These configurations are easily modified to have contiguous sum co-arrays and $R_{\infty}=2$ for any $\rho$. It is therefore interesting to compare the CRA to these solutions when $\rho \neq 1$. An appropriate quantity for this is the asymptotic ratio of the number of elements in the CRA to the two aforementioned arrays. The element redundancy $\eta_{\infty}=$ $\sqrt{R_{\infty} / 2}=(\rho+1) /(2 \sqrt{\rho})$ shows that the CRA is only slightly inefficient for moderate $\rho$. E.g. $\rho=0.5$ yields $\eta_{\infty} \approx 1.06$, which implies that large CRAs require at most $6 \%$ more elements than the two aspect-ratio-independent configurations, when $0.5 \leq \rho \leq 1$. Non-square arrays are relevant in many practical applications, such as radar and wireless communications that may require different resolutions in azimuth and elevation.

\section{IMAGING EXAMPLE WITH MUTUAL COUPLING}

This section compares the imaging performance of the URA, BA and CRA in the presence of mutual coupling, which is not compensated for in any manner. An array aperture of $12 \times 12$ unit spacings is chosen, leading to $N=48$ elements in the BA and CRA (Fig. 3), and $N=169$ in the URA. Elements are assumed identical, reciprocal, and omnidirectional. Entries of the coupling matrix are given by $M_{n m}=c_{1} e^{j \phi l_{n m}} / l_{n m}$, where $l_{n m}=\left\|\mathbf{d}_{n}-\mathbf{d}_{m}\right\|_{2} /(\lambda / 2)$ is the Euclidean distance between elements $n$ and $m$ in units of the inter-element spacing $\lambda / 2$.

A scene consisting of $K=16$ far-field, unit reflectivity $\left(\gamma_{k}=1, \forall k\right)$ point targets is imaged. Target azimuth and elevation are given by $\varphi_{k} \in\{ \pm 3, \pm 1\} \cdot \pi / 10$ and $\theta_{k} \in$ $\{1,2,3,4\} \cdot \pi / 5$. The coupling magnitude is $c_{1}=0.2$, and the coupling phase is a uniformly distributed random variable, $\phi \sim \operatorname{Uni}(0,2 \pi)$. The image RMSE is $\varepsilon=\left\|\mathbf{Y}_{\mathrm{d}}-\alpha \mathbf{Y}\right\|_{\mathrm{F}} / \sqrt{N_{\varphi} N_{\theta}}$, where $\mathbf{Y}, \mathbf{Y}_{\mathrm{d}} \in \mathbb{C}^{N_{\varphi} \times N_{\theta}}$ are the beamformed images with and without mutual coupling, and $N_{\varphi}=N_{\theta}=201$ are the number of scanned azimuth and elevation angles. Furthermore, $\alpha=\operatorname{Tr}\left(\mathbf{Y}^{\mathrm{H}} \mathbf{Y}_{\mathrm{d}}\right) /\|\mathbf{Y}\|_{\mathrm{F}}^{2}$ is the scaling factor minimizing $\varepsilon$. A pixel of the image assumes the form $Y_{i j}=\sum_{q=1}^{Q} \mathbf{w}_{\mathrm{r}}^{(q)}{ }^{\mathrm{H}} \mathrm{Xw}_{\mathbf{t}}^{(q)}$. Here $Q$ is number of component images, which is chosen such that $99.99 \%$ of the variation in the desired PSF (under the coupling-free model) is captured by the set of $\mathrm{Tx}$ and $\mathrm{Rx}$ weights $\left\{\mathbf{w}_{\mathrm{t}}^{(q)}, \mathbf{w}_{\mathrm{r}}^{(q)}\right\}_{q=1}^{Q}$ [34]. These weights are computed using SVD image addition [20] with low-rank matrix recovery [34]. The desired co-array weighting is set to a 2D DolphChebyshev window [35] with $-40 \mathrm{~dB}$ sidelobes.

Fig. 5 shows the magnitude of images $\mathbf{Y}_{\mathrm{d}}$ (URA, couplingfree) and $\alpha \mathbf{Y}$ (URA, BA, and CRA; coupling phase $\phi=$ $1.15 \pi)$. The sample mean $\hat{\mu}$ and standard deviation $\hat{\sigma}$ of the RMSE are also displayed (100 realizations of $\phi$ ). The CRA achieves $\hat{\mu} \pm \hat{\sigma} \approx(6 \pm 0.8) \cdot 10^{-2}$, which is approximately $25 \%$ lower than the BA with $(8 \pm 1) \cdot 10^{-2}$. However, the visual difference between the two images is negligible. The URA has the lowest side lobes, but the highest RMSE $(13 \pm 3) \cdot 10^{-2}$, since targets appear weaker closer to boresight than endfire.

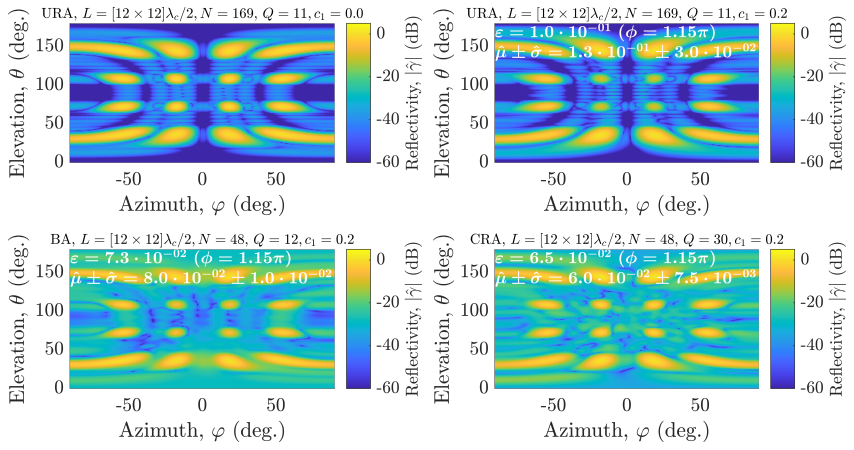

Fig. 5. With image addition and no mutual coupling, the same image (top left) is achieved by all three arrays. With mutual coupling $\left(c_{1}=0.2, \phi=1.15 \pi\right)$, the CRA (bottom right) achieves lower RMSE $\varepsilon$ than the URA (top right) and BA (bottom left), in addition to lower average RMSE $\hat{\mu} \pm \hat{\sigma}$ (100 random $\phi)$.

In reality, sparseness alone does not explain the extent of mutual coupling experienced by an array. In fact, the CRA does not always outperform the BA and URA, even in simulations using the simple signal model of (1). Results depend on, e.g., the target scene $\boldsymbol{\Gamma}$, coupling parameters $\left(c_{1}, \phi\right)$, and scan range of $(\varphi, \theta)$. Additionally, the periodic structure of the BA (and obviously the URA) could be leveraged in simplifying mutual coupling compensation or performance analysis [36], [37]. Periodicity also enables the URA to avoid scan blindness when elements are spaced closer than $\lambda / 2$ [38]. Therefore, a more thorough comparison of sparse active arrays in the presence of mutual coupling is needed. This is however beyond of the scope of this signal processing letter and left for future work.

\section{CONCLUSIONS}

This paper introduced the Concentric Rectangular Array (CRA) and established its key properties. The CRA is a sparse active array with transceiving elements confined to a rectangular area. The array has a contiguous sum and difference co-array, and the same number of elements as the Boundary Array (BA). Both of these arrays are actually MinimumRedundancy Arrays (MRAs) for certain square arrays. In some cases, the CRA is the MRA with the fewest unit spacings. The CRA and BA are generally not MRAs for non-square apertures. However, large CRAs with aspect ratio $\rho \geq 0.5$ require $\leq 6 \%$ more elements than currently known aspect-ratioindependent configurations. Furthermore, the number of unit element displacements in the CRA remains low and practically independent of aperture, which in some cases makes it less susceptible to the effects of mutual coupling. 


\section{REFERENCES}

[1] R. A. Haubrich, "Array design," Bulletin of the Seismological Society of America, vol. 58, no. 3, pp. 977-991, 1968.

[2] R. T. Hoctor and S. A. Kassam, "High resolution coherent source location using transmit/receive arrays," IEEE Transactions on Image Processing, vol. 1, no. 1, pp. 88-100, Jan 1992.

[3] P. Pal and P. P. Vaidyanathan, "Nested arrays: A novel approach to array processing with enhanced degrees of freedom," IEEE Transactions on Signal Processing, vol. 58, no. 8, pp. 4167-4181, Aug 2010.

[4] A. Koochakzadeh and P. Pal, "Cramér-Rao bounds for underdetermined source localization," IEEE Signal Processing Letters, vol. 23, no. 7, pp. 919-923, July 2016.

[5] C. L. Liu and P. Vaidyanathan, "Cramér-Rao bounds for coprime and other sparse arrays, which find more sources than sensors," Digital Signal Processing, vol. 61, pp. 43 - 61, Feb 2017.

[6] M. Wang and A. Nehorai, "Coarrays, MUSIC, and the Cramér-Rao bound," IEEE Transactions on Signal Processing, vol. 65, no. 4, pp. 933-946, Feb 2017.

[7] R. T. Hoctor and S. A. Kassam, "The unifying role of the coarray in aperture synthesis for coherent and incoherent imaging," Proceedings of the IEEE, vol. 78, no. 4, pp. 735-752, Apr 1990.

[8] E. BouDaher, F. Ahmad, M. G. Amin, and A. Hoorfar, "Mutual coupling effect and compensation in non-uniform arrays for direction-of-arrival estimation," Digital Signal Processing, vol. 61, pp. 3-14, 2017.

[9] C. L. Liu and P. P. Vaidyanathan, "Super nested arrays: Linear sparse arrays with reduced mutual coupling - part I: Fundamentals," IEEE Transactions on Signal Processing, vol. 64, no. 15, pp. 3997-4012, Aug 2016.

[10] _ - "Hourglass arrays and other novel 2-d sparse arrays with reduced mutual coupling," IEEE Transactions on Signal Processing, vol. 65, no. 13, pp. 3369-3383, July 2017.

[11] I. Gupta and A. Ksienski, "Effect of mutual coupling on the performance of adaptive arrays," IEEE Transactions on Antennas and Propagation, vol. 31, no. 5, pp. 785-791, Sep 1983.

[12] B. Friedlander and A. J. Weiss, "Direction finding in the presence of mutual coupling," IEEE Transactions on Antennas and Propagation, vol. 39, no. 3, pp. 273-284, Mar 1991.

[13] B. Wichmann, "A note on restricted difference bases," Journal of the London Mathematical Society, vol. s1-38, no. 1, pp. 465-466, 1963.

[14] D. Pearson, S. U. Pillai, and Y. Lee, "An algorithm for near-optimal placement of sensor elements," IEEE Transactions on Information Theory, vol. 36, no. 6, pp. 1280-1284, Nov 1990.

[15] D. A. Linebarger, I. H. Sudborough, and I. G. Tollis, "Difference bases and sparse sensor arrays," IEEE Transactions on Information Theory, vol. 39, no. 2, pp. 716-721, Mar 1993.

[16] P. P. Vaidyanathan and P. Pal, "Sparse sensing with co-prime samplers and arrays," IEEE Transactions on Signal Processing, vol. 59, no. 2, pp. 573-586, Feb 2011.

[17] R. Rajamäki and V. Koivunen, "Sparse linear nested array for active sensing," in 25th European Signal Processing Conference (EUSIPCO 2017), Kos, Greece, August 2017.

[18] _ _ "Symmetric sparse linear array for active imaging," in Tenth IEEE Sensor Array and Multichannel Signal Processing Workshop (SAM 2018), Sheffield, UK, July 2018, pp. 46-50.

[19] G. R. Lockwood, P.-C. Li, M. O'Donnell, and F. S. Foster, "Optimizing the radiation pattern of sparse periodic linear arrays," IEEE Transactions on Ultrasonics, Ferroelectrics, and Frequency Control, vol. 43, no. 1 , pp. 7-14, Jan 1996.

[20] R. J. Kozick and S. A. Kassam, "Linear imaging with sensor arrays on convex polygonal boundaries," IEEE Transactions on Systems, Man, and Cybernetics, vol. 21, no. 5, pp. 1155-1166, Sep 1991.

[21] J. Kohonen, V. Koivunen, and R. Rajamäki, "Planar additive bases for rectangles," arXiv preprint arXiv:1711.08812, 2017.
[22] T. Su and H. Ling, "On modeling mutual coupling in antenna arrays using the coupling matrix," Microwave and Optical Technology Letters, vol. 28, no. 4, pp. 231-237, 2001.

[23] M. Wang, Z. Zhang, and A. Nehorai, "Performance analysis of coarraybased MUSIC in the presence of sensor location errors," IEEE Transactions on Signal Processing, vol. 66, no. 12, pp. 3074-3085, June 2018.

[24] R. J. Kozick and S. A. Kassam, "Synthetic aperture pulse-echo imaging with rectangular boundary arrays," IEEE Transactions on Image Processing, vol. 2, no. 1, pp. 68-79, Jan 1993.

[25] F. Ahmad and S. A. Kassam, "Coarray analysis of the wide-band point spread function for active array imaging," Signal Processing, vol. 81, no. 1, pp. 99-115, 2001.

[26] F. Ahmad, G. J. Frazer, S. A. Kassam, and M. G. Amin, "Design and implementation of near-field, wideband synthetic aperture beamformers," IEEE Transactions on Aerospace and Electronic Systems, vol. 40, no. 1, pp. 206-220, Jan 2004

[27] C. M. Coviello, R. J. Kozick, A. Hurrell, P. P. Smith, and C. C. Coussios, "Thin-film sparse boundary array design for passive acoustic mapping during ultrasound therapy," IEEE Transactions on Ultrasonics, Ferroelectrics, and Frequency Control, vol. 59, no. 10, October 2012.

[28] R. T. Hoctor and S. A. Kassam, "Array redundancy for active line arrays," IEEE Transactions on Image Processing, vol. 5, no. 7, pp. 1179-1183, Jul 1996.

[29] G. Yu, "Upper bounds for finite additive 2-bases," Proceedings of the American Mathematical Society, vol. 137, no. 1, pp. 11-18, 2009.

[30] A. Moffet, "Minimum-redundancy linear arrays," IEEE Transactions on Antennas and Propagation, vol. 16, no. 2, pp. 172-175, Mar 1968.

[31] J. Kohonen, "A meet-in-the-middle algorithm for finding extremal restricted additive 2-bases," Journal of Integer Sequences, vol. 17, no. 6, 2014.

[32] M. F. Challis, "Two new techniques for computing extremal h-bases ak," The Computer Journal, vol. 36, no. 2, pp. 117-126, 1993.

[33] C. L. Liu and P. P. Vaidyanathan, "Maximally economic sparse arrays and Cantor arrays," in Seventh IEEE International Workshop on Computational Advances in Multi-Sensor Adaptive Processing (CAMSAP 2017), Dec 2017, pp. 1-5.

[34] R. Rajamäki and V. Koivunen, "Sparse array imaging using low-rank matrix recovery," in Seventh IEEE International Workshop on Computational Advances in Multi-Sensor Adaptive Processing (CAMSAP 2017), Curaçao, Dutch Antilles, December 2017.

[35] C. L. Dolph, "A current distribution for broadside arrays which optimizes the relationship between beam width and side-lobe level," Proceedings of the IRE, vol. 34, no. 6, pp. 335-348, June 1946.

[36] J. Rubio, J. F. Izquierdo, and J. Córcoles, "Mutual coupling compensation matrices for transmitting and receiving arrays," IEEE Transactions on Antennas and Propagation, vol. 63, no. 2, pp. 839-843, Feb 2015.

[37] D. M. Pozar, "The active element pattern," IEEE Transactions on Antennas and Propagation, vol. 42, no. 8, pp. 1176-1178, Aug 1994.

[38] J. L. Allen and B. L. Diamond, "Mutual coupling in array antennas," Massachusetts Institute Of Technology Lexington Lincoln Lab, Tech. Rep., 1966. 